\title{
Comprehensive Study of the Benefits of Integrating a Sharing Energy Strategy between Prosumers
}

\author{
M. Ruiz-Cortés, E. Romero-Cadaval, C. Roncero-Clemente, F. Barrero-González, E. González-Romera \\ Power Electrical and Electronic Systems (PE\&ES) \\ University of Extremadura \\ Badajoz, Spain
}

\begin{abstract}
The upgrade of power systems in the last years due to the proliferation of distributed generation with renewable energy sources, active users and electric vehicles has become a challenge. In this new scenario, prosumers play an important role in order to buffer the consumption curve and, therefore, contributing to the grid requirements. Special attention is attracted by photovoltaic (PV) arrays integrated in residential buildings, because they can be complemented with energy storage systems (ESSs), such as batteries or ultracapacitors (UCs) or even a combination of both, with the aim to achieve that customers could manage their energy. The above reasons justify the need of an energy management system (EMS) that coordinates the operation of the energy storage devices in an optimized way, as they are expensive. In this paper, a comprehensive control system is proposed to exchange energy between the hybrid storage devices (batteries and UCs) of two dwells with PV installations in order to increase the availability of their ESSs and support the energy requirements during the most time possible, optimizing sizes and costs and avoiding saturation.
\end{abstract}

Keywords-prosumer; photovoltaic system; energy storage system; battery; ultracapacitor; energy management; sharing energy strategy

\section{INTRODUCTION}

Nowadays, power systems have to face multiple challenges as a consequence of the apparition of distributed generation [1] in that renewable energy sources, active users and electric vehicles have a fundamental role. This is because of they cause technical problems in voltage and frequency regulation, islanding, harmonic distortion, electromagnetic interference or even distribution issues [2]. With the aim of mitigating these problems and ensuring the safety and reliability of the power system, a new concept about the role of end users is required: end users are called to be prosumers.

In this new scenario, users can be producers, consumers or prosumers. A prosumer is a combination of a producer and a consumer, and it can be defined as a user able both to extract power from the grid and to deliver power into the grid [3]. Prosumers combine loads which have to be fed and energy sources that provide supply according to economic decisions. If the number of prosumers is high, they can contribute to smooth the demand curve, and, therefore, to reduce the required power generation. Moreover, prosumers equipped with control and communication systems can interact with the main grid providing services such as the increase of the penetration of intermittent renewable energy sources into the power system, the load/generation shifting, ancillary services and grid system applications [3], [4]. As a result, several benefits can be achieved: the decrease both the needed peak power generation and the charge on the electric grid, and also a reduction of the usage of fossil fuels [5]. On the other hand, end users could optimize the utilization of renewable energy sources and achieve lower electricity bills [4].

In recent years, PV sources have experimented a great development [6] and have become the most common renewable energy source present in residential and commercial customer properties connected to low voltage distribution networks [2], [7]. The PV installations development has been particularly significant in urban areas, where these small power plants are associated or integrated to buildings [8]. This fact can be explained taking into account that PV systems can be installed on the roof of buildings instead of occupying large land areas, and owners can benefit from electricity cost savings [2].

However, PV systems in residential applications present some problems: there is a mismatch between the peak PV power generation time, which is usually produced at noon, and the peak load power consumption, which is usually produced in the evening [5]. Moreover, PV power generation presents an intermittent and unpredictable character as a consequence to its dependence of the solar irradiance. An effective solution to the previous issues which has been widely accepted in the technical literature consists in incorporating an ESS in the buildings with PV installation. The function of the proposed residential ESS is to store the excess of power from the PV system during noon time and thus reduce the energy loss associated with the surplus power from the solar PV array [7] and decrease the injection in the grid from the PV system [1]. This stored energy could be used later to feed the load when PV supply is not sufficient, serving as support to the grid. Therefore, ESSs allow the displacement of the energy consumption of customers, in a way that they only inject when the energy is needed most and charge their ESSs when there is an excess of generation or the price of the energy is cheaper than in other periods [1]. Consequently, ESSs contribute to smooth the load consumption curve and improve the energy management [1].

There are several technologies proposed to be combined with PV systems, such as batteries (which are the most 


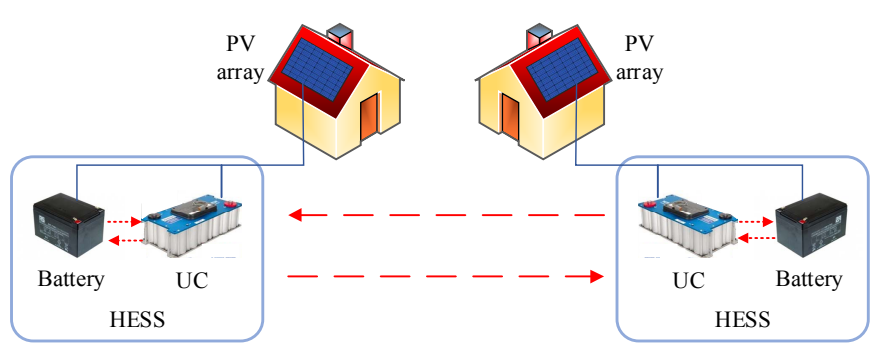

Fig. 1. Case study scenario.

commonly used), UCs or even fuel cells [9]. In the design of the employed ESS is essential to decide what of these technologies is the most suitable for the application [10]. In the case of PV generation, it is important taking into account that $\mathrm{PV}$ is composed by a low-frequency component and highfrequency oscillations associated to the intermittency of the solar irradiance. Thus, in order to smooth the low-frequency fluctuations, an ESS with high energy density is required, while an ESS with high power density is required to provide or absorb the high-power transients [11].

Between the different ESS technologies, batteries are able to store acceptable amounts of energy, but they cannot deliver large amount of energy in a very short time [12]. On the other hand, UCs can supply high peaks of energy and can be charged and discharged without missing of efficiency for thousands of cycles, although their storage capacity is low [12]. Both technologies can be combined to add the high energy density of batteries to the high-power density of UCs [10]-[13]. Besides, the use of UCs in cooperation with batteries can contribute to improve batteries lifetime because UCs are responsible for provide high power peaks, which can damage batteries [13]. This combination of ESS technologies makes up a hybrid energy storage system (HESS) [13].

Different HESS topologies exist. However, one of the most used HESS topologies is the parallel configuration of the battery/UC HESS with bidirectional converters. The use of bidirectional converters allows the management of each ESS separately and even the exchange of the energy between the ESSs [13], providing more flexibility.

HESS must work coordinately with PV system, but a coordination between the storage devices that conform the HESS must exist also, with the aim of they can interact between themselves and, thus, an optimized operation of the overall system is achieved. For this purpose, it is necessary an energy management system (EMS) that regulates the energy that HESS stores/supplies according to generation/consumption conditions and the energy that is assumed by each device of the HESS. Moreover, EMS can incorporate an exchanging energy cooperative strategy between the storage devices that ensures their availability. For example, the batteries can charge and discharge the UCs, which is very useful to guarantee that UCs do not suffer saturation and are available to support batteries at all times. EMS could integrate also a sharing energy strategy between prosumers connected to the same point of common coupling (PCC), that means that prosumers can establish energy exchanging relationships between themselves for regulating the charge of their storage systems, increasing the use of their batteries and minimizing their dependence on the distribution grid. In this scenario, prosumers which supply energy to other prosumers could lead additional economic benefits, and communities of prosumers as autonomous as possible of the main grid could be created.

This paper presents a simulation case study with the aim of analyzing the behavior of two prosumer dwells which have a PV array and a battery/UC HESS through their power generation and consumption curves, as it is described in Section II. The installations of these prosumers are managed with the sharing energy strategy exposed in Section III. The objective of the proposed EMS is to maintain UCs voltage in a desired level within each HESS and to exchanged energy between the batteries of both dwells, as it is described in Section III in more detail. In this way, the EMS facilitates, on one hand, the optimization of the HESS operation of each prosumer and, on the other hand, the cooperation between prosumers connected to the same PCC in order to manage the SOC of their batteries. In Section IV, simulation results obtained for the case study are discussed in order to evaluate if the proposed control strategy is valid. Finally, in Section V, this paper finishes with a brief explanation about the main conclusions which have been obtained in this work.

\section{CASE STUDY}

Fig. 1. depicts the analyzed case study. It represents two residential prosumers connected to a low voltage distribution network at the same PCC. Each residential prosumer has a small PV system and a HESS based on the combination of lead-acid batteries and UCs. Lead-acid batteries have been chosen because of their higher economic feasibility in comparison with other technologies). The peak PV powers are $3 \mathrm{kWp}$ in the case of prosumer 1 and $5 \mathrm{kWp}$ in the case of prosumer 2. HESSs have been dimensioned in order to batteries have three days of autonomy [14]. The characteristics of each PV array and each HESS are collected in Table I. Besides, the typical diary load consumption and PV generation profiles of prosumer 1 and prosumer 2 are shown in Fig. 2 and Fig. 3 respectively. These curves have been obtained through the simulation of provided data using Simulink/Matlab software.

TABLE I. SIMULATION PARAMETERS

\begin{tabular}{|l|c|c|}
\hline \multirow{2}{*}{\multicolumn{1}{|c|}{ Parameter }} & \multicolumn{2}{c|}{ Value } \\
\cline { 2 - 3 } & Prosumer 1 & Prosumer 2 \\
\hline Peak PV power & $3 \mathrm{kWp}$ & $5 \mathrm{kWp}$ \\
\hline Batteries bank capacity & $2000 \mathrm{Ah}$ & $3100 \mathrm{Ah}$ \\
\hline UCs bank capacity & $600 \mathrm{~F}$ & $900 \mathrm{~F}$ \\
\hline HESS devices voltage & $48 \mathrm{~V}$ & $48 \mathrm{~V}$ \\
\hline
\end{tabular}




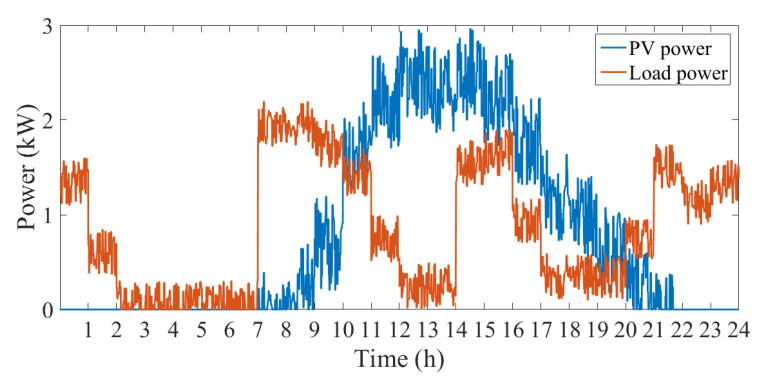

Fig. 2. PV generation and load consumption curves of prosumer 1.

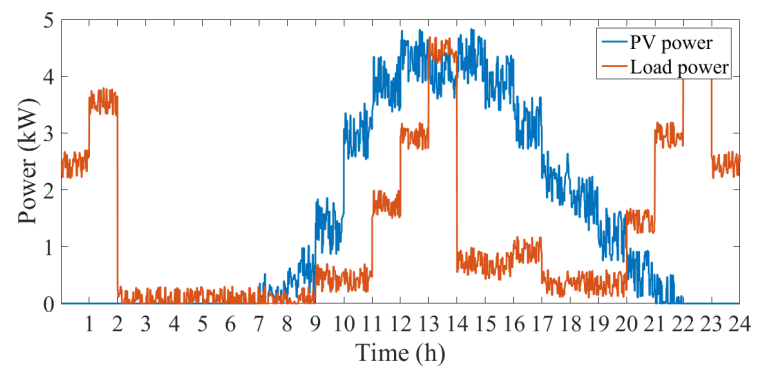

Fig. 3. PV generation and load consumption curves of prosumer 2.

\section{PROPOSED CONTROL STRATEGY}

An EMS is needed in order to HESS works properly. As we mentioned above, batteries are suitable to provide the bulk of energy during large intervals of time, whereas UCs are responsible for supplying the peak power demands and absorbing the peak PV power excess. This can be achieved using a conventional low-pass filter [11], that separates the low-frequency component of the power assumed by the HESS, which is destined to the batteries, and the high-frequency component, which is took on the UCs. The characteristics of the low-pass filter have been designed taking into account the considerations of [15]. This power sharing is represented by $(1),(2)$ and (3), where the power that have to be provided by the HESS $\left(P_{\text {ref,sto }}\right)$ is calculate as the difference between the demanded power $\left(P_{\text {load }}\right)$ and the PV generated power $\left(P_{P V}\right)$, whereas $P_{b a t}$ and $P_{U C}$ are the power assumed by the battery bank and the UC bank respectively. Fig. 4 shows the block diagram built in Simulink in order to model the below equations.

$$
\begin{gathered}
P_{\text {ref,sto }}=P_{\text {load }}-P_{P V} \\
P_{b a t}=\operatorname{LPF}\left(P_{\text {ref,sto }}\right) \\
P_{U C}=P_{r e f, s t o}-P_{b a t}
\end{gathered}
$$

An important aspect to have into account is the state of charge (SOC) of the batteries. In [16], the capacity of the battery is defined as the quantity of electrical charge in the cell from the fully charged state to the discharged state. Thus, the

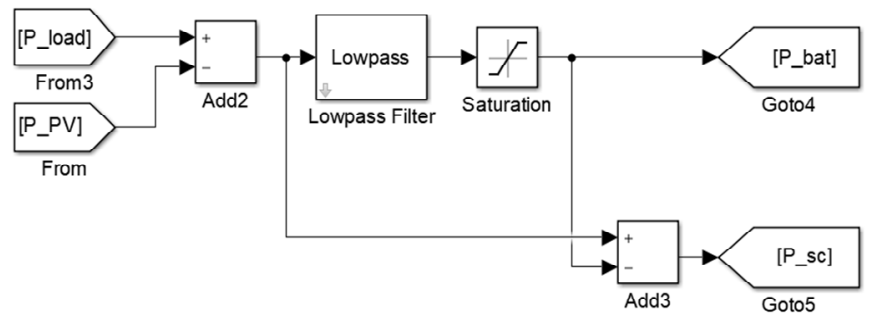

Fig. 4. Simulink scheme used to calculate $P_{b a t}$ and $P_{U C}$.

SOC means the ratio between the remaining capacity and the full charge; hence, a SOC equal to $100 \%$ implies that the battery is fully charged, whereas a SOC equal to $0 \%$ indicates that the battery is fully discharged [16]. It is recommended that batteries work within a determined working range, because of working out of this range could cause a loss of battery life [17]. For this reason, a limitation of the SOC of the batteries has been considered in this paper. In this way, batteries are able to work only within a range whose upper limit has been established in a value of $100 \%$ and the lower limit is equal to $20 \%$, according to lead-acid batteries characteristics [14]. Thereby, it is possible to avoid over-charging and overdischarging in the batteries.

Furthermore, the EMS is key to a cooperation between the batteries and the UCs banks happens [13]. This cooperation is based on the need of maintaining the voltage in the UCs system in a certain level [11], which has been established in the $50 \%$ of its maximum value. In this way, when the UC voltage is greater than the reference value, the EMS generates a current reference through a PI controller to charge the batteries from the UCs bank until the UCs bank reaches its voltage reference value. Thanks to this control action, the UCs remain at every moment able to provide energy (positive during discharging cycles or negative during charging cycles), and it is possible to avoid that they suffer high charge fluctuations. The reason to adopt an UC voltage reference value equal to $50 \%$ is explained in [18] and is based on (4) and (5), where $S O C_{U C}$ and $V$ are the SOC and the voltage of the UC in a certain instant respectively, $V_{\text {nom }}$ is the UC rated voltage and $C$ is the UC capacity,

$$
\begin{aligned}
& S O C_{U C}=\frac{V}{V_{n o m}} \\
& E_{U C}=\frac{1}{2} \cdot C \cdot V^{2} .
\end{aligned}
$$

As we can see in (5), an UC stores energy proportionally to the square of its voltage. Thus, when $S O C_{U C}$ reaches $50 \%$, it means that $75 \%$ of UC stored energy has been consumed [18]. Because of this, we have considered that the UCs bank voltage must be maintained in a minimum value of $50 \%$ of its maximum value with the aim of the UCs are available to provide its corresponding power. Additional considerations can be found in [19]. 

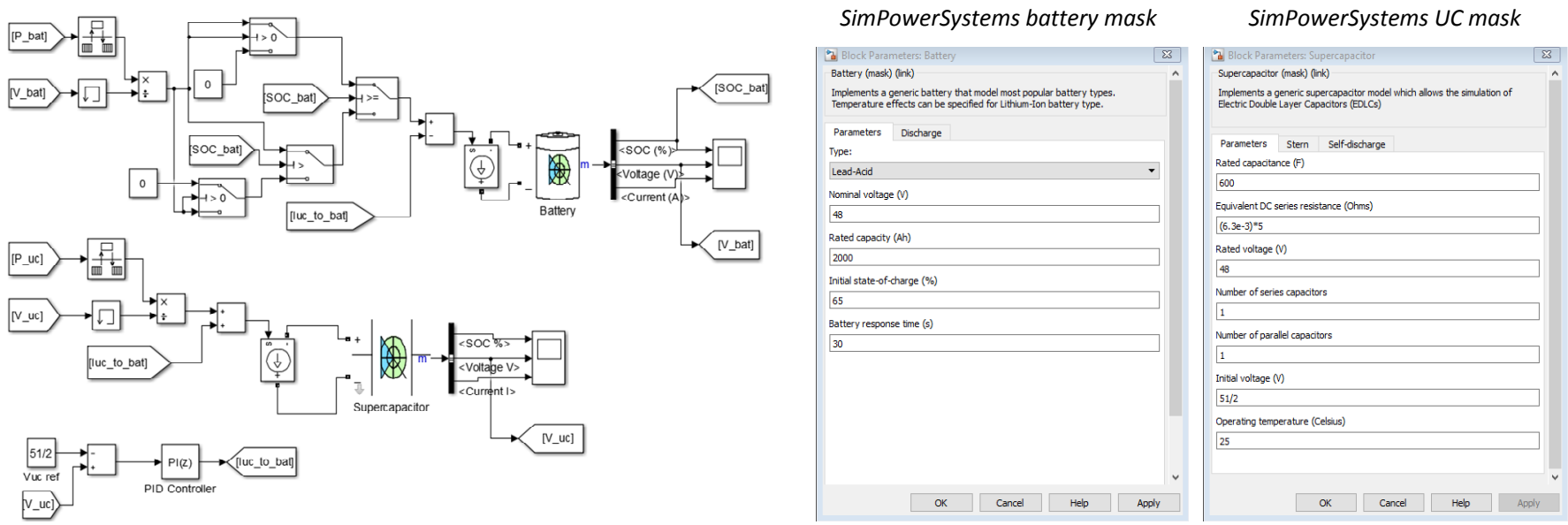

Fig. 5. Simulink model used to simulate the battery and UC banks work with the proposed control.

Fig. 5 shows the model built in Simulink for simulating the HESS of each one of the analyzed prosumers and its energy management. As we can see in this figure, we have used the battery and UC models that Simulink provides in SimPowerSystems Blockset, and they work through controlled current sources.

However, the action of the EMS is not only limited to exchange energy between the storage devices of the same HESS, since it is possible use it to share energy between different users. This idea has been added to the EMS proposed in this paper in the following way: when the SOC of one of the batteries is low, it is helped by the battery of the other

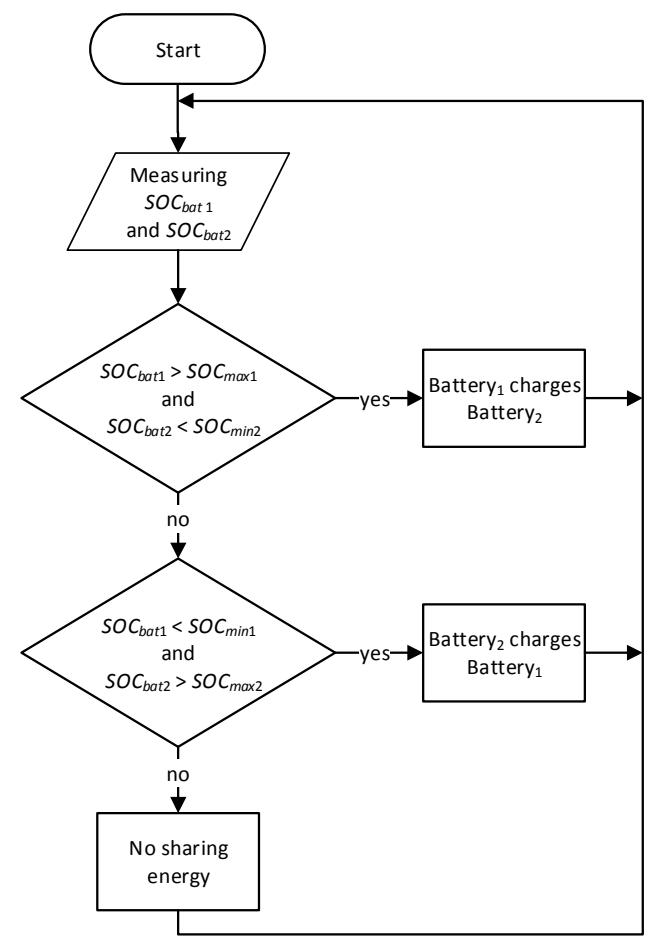

Fig. 6. Flow chart of the sharing energy strategy between batteries. prosumer to provide energy and, therefore, to contribute to increase the SOC of the first battery. So as to guarantee a suitable behavior of the two simulated HESSs, certain constraints have to be considered. These restrictions consist of a battery only can provide energy to the other battery if its SOC is greater than a maximum value $\left(S O C_{\max }\right)$, whereas a battery SOC must be lower than a minimum value $\left(S O C_{\min }\right)$ to be charged by the battery with high SOC (Fig. 6). The values of $S O C_{\max }$ and $S O C_{\min }$ depends on the type of battery and the characteristics of the installation. In this way, we can avoid, on one hand, that the battery which shares energy with the other suffers an excessive discharge, and, on the other hand, that the battery which receives energy is not available when the battery of a neighbor is sufficient charged or even overcharged. This strategy leads to prosumers can manage the SOC of their batteries keeping it in a medium level with the purpose of both batteries are available to supply/store the required energy during the most time possible, avoiding saturation in the batteries ( $\mathrm{SOC} \leq 20 \%$ or $\mathrm{SOC}=100 \%$ ) and, therefore, their disconnection of the prosumer installation. In addition, it draws on the stored energy of the battery with more SOC while its owner does not need it.

Fig. 7. shows the block diagram built in Simulink to implement this control action in the below model.

\section{Simulation Results}

\section{A. Energy Exchange in an Individual HESS}

The proposed control strategy has been implemented by simulation in the HESSs of the depicted dwells to manage energy between the battery and the UC systems of each customer. As we can see in Fig. 8, the control strategy accomplishes the requirement of avoiding UCs suffer saturation when their SOC reaches $100 \%$ and also a fully discharge. This has been achieved through the exchange of energy between the batteries and the UCs banks. Thus, the SOC of the battery has been affected in the way shown in Fig. 9, which compares the SOC of the batteries bank when the proposed EMS is used with the case in that it is not applied. In the same figure, it can be observed how the batteries bank 


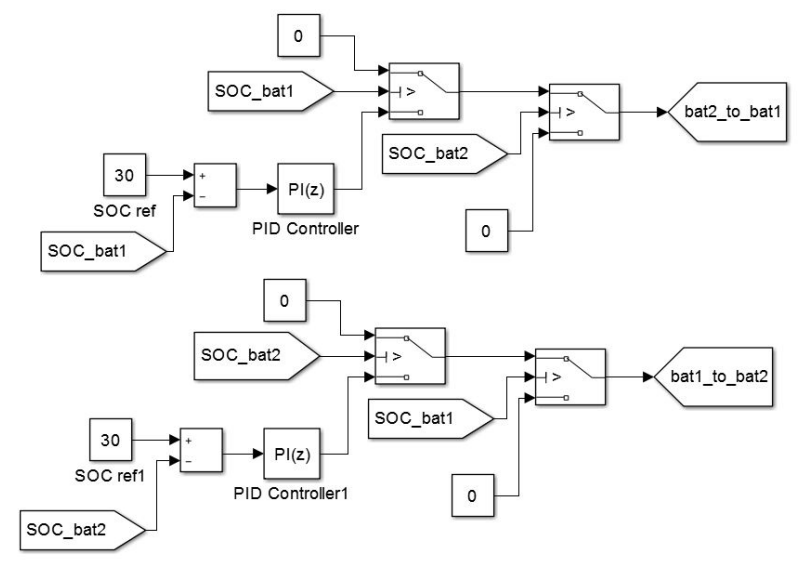

Fig. 7. Simulink block diagram used to implement the energy sharing between prosumers.

provides and absorbs energy to the UCs when it is necessary in order to the UCs bank voltage remains in the desired value. However, the difference between both SOC curves is small.

On the other hand, Fig. 10 shows the current that the battery and the UC supply respectively during an interval of one hour, corresponding to the period contained between 8:00 am and 9:00 am, in the case of the prosumer 1. In this figure, it can be proved how the battery delivers the low-frequency component of the needed current, whereas the UC provides the required high-frequency component of the current to operate properly with the PV system (positive currents imply discharge in the ESSs and negative currents mean charge). Simulation results are similar in the case of the prosumer 2.

\section{B. Energy cooperation between prosumers}

Besides, the sharing energy between prosumers has been integrated in the control strategy. In this work, the limits of batteries for sharing energy have been set at $S O C_{\min }=30 \%$ and $S O C_{\max }=60 \%$, considering the same values in both batteries. It has been simulated in the following conditions: the initial SOC of the battery of prosumer 1 is equal to $25 \%$ and the initial SOC of the battery of prosumer 2 is $75 \%$. Therefore, the battery of the prosumer 2 has to cooperate with the battery of prosumer 1, as it is shown in Fig. 11. As we can see in this figure, sharing energy strategy achieves SOC of the battery of prosumer 1 improves, increasing until reaching the 30\%, which is the established limit, at 5:00 am approximately. As a consequence, during this interval, the SOC of the battery of prosumer 2 decreases in order to provide energy to prosumer 1, and also from 7:00 am to 10:00 am, when the SOC of the battery of prosumer 1 drops under $30 \%$, and accomplishes to rise it while the energy required by prosumer 2 is delivered also. The rest of the time both batteries do not exchange energy between them and they are charged and discharged according to the PV generation and the load consumption.

\section{CONCLUSIONS}

This paper exposes the relevance that prosumers have reached in the last years into the new scenario that the development of distributed generation has caused. Particularly,

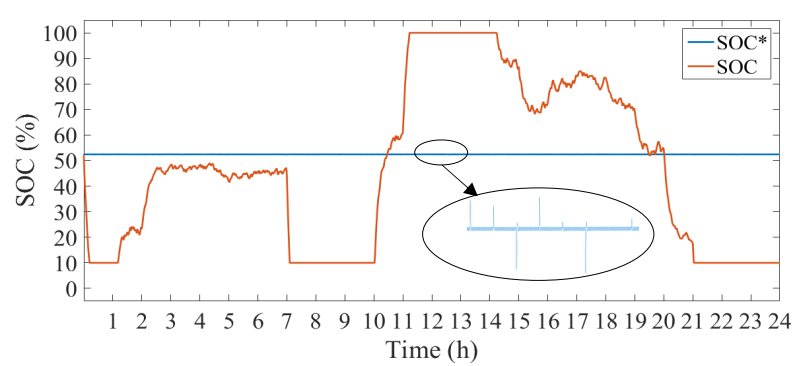

Fig. 8. Comparison of the evolution of the SOC of the UC system along a day with the proposed control action (marked with *) and without it.

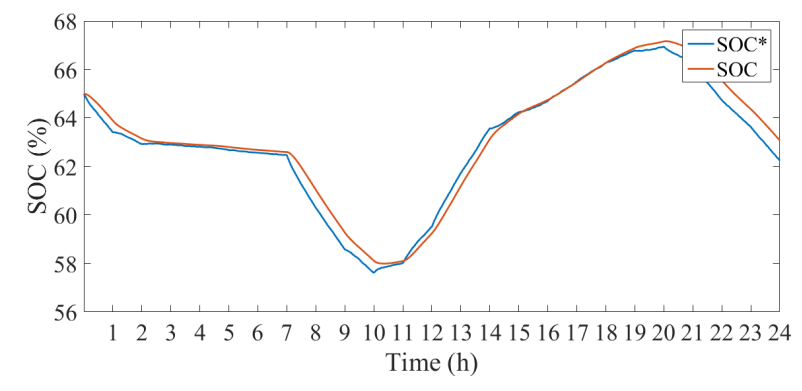

Fig. 9. Comparison of the evolution of the SOC of the battery with the control strategy (SOC*) and without it (SOC).

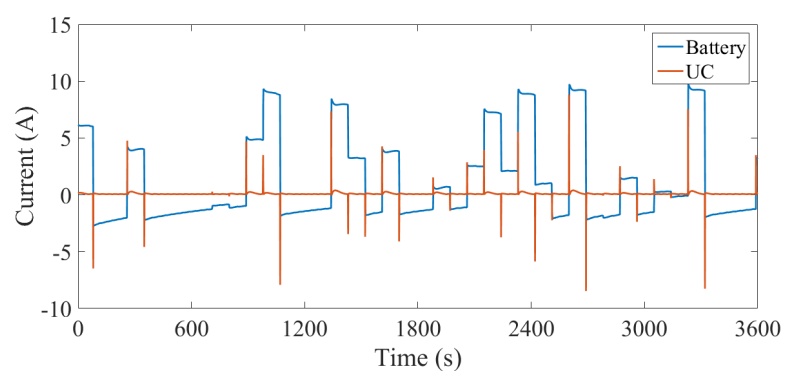

Fig. 10. Comparison of the currents provided by the battery and the UC systems since 8:00 am to 9:00 am in the case of the prosumer 1 .

the case of residential prosumer installations with a small PV array combined with a HESS based on batteries and UCs has been studied by simulation. In addition, a comprehensive control strategy has been proposed in order to optimize the energy management.

This energy management allows to maintain the voltage of the UCs bank of a HESS in a medium value, which guarantees that UCs will always be ready to absorb or inject power peaks. A similar approach is the base of the sharing energy system integrated in order to prosumers can cooperate together exchanging energy between their batteries according to their respective states of charge. In this way, it is possible to avoid one of them is not able to supply energy whereas the other is enough charged to provide its corresponding energy reference and to serve as support to the battery with low SOC simultaneously. Thus, this strategy prevents from saturating batteries caused by over-charging and over-discharging, achieving a greater use of the capacity of the costly ESSs and a maximization of the PV generation, due to it is not necessary to change the MPPT in order to decrease the generation because of batteries are saturated and cannot store more energy. 


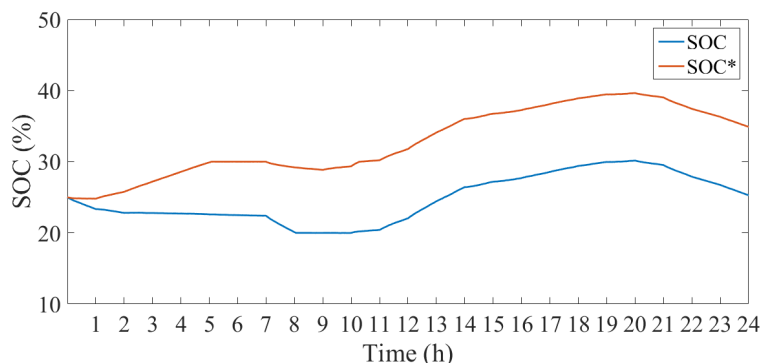

(a)

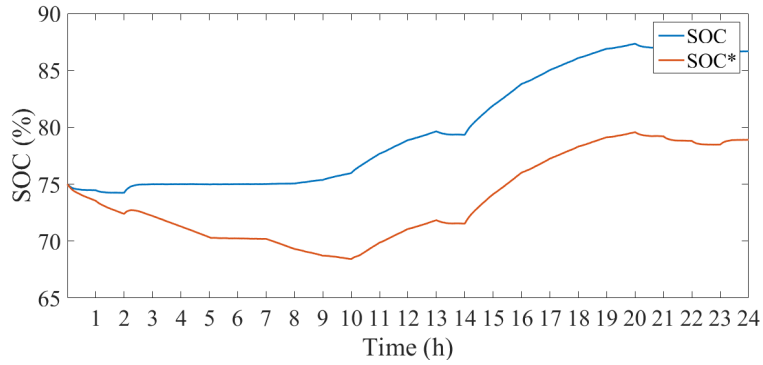

(b)

Fig. 11. Comparison of the evolution of the SOC of the batteries of prosumer 1 (a) and prosumer 2 (b) without sharing energy strategy (SOC) and with it (SOC*).

Therefore, this sharing energy strategy could serve to optimize the capacity sizing of ESSs and PV arrays.

In this paper, the sharing energy between batteries of different prosumers is realized only during determined periods of time along the day in order to avoid a prosumer cannot feed his loads without the support of the main grid, but a continuous sharing energy strategy could also be done with the aim of balancing the batteries of both prosumers, which is the next step to this work.

\section{ACKNOWLEDGMENT}

This research work has been partially supported by the project (TEC2016-77632-C3-1-R) from "Ministerio de Economía y Competitividad (Gobierno de España)" and by "Junta de Extremadura" with funds for research groups (GR15177).

\section{REFERENCES}

[1] S. M. Souza, M. Gil, J. Sumaili, A. G. Madureira and J. A. P. Lopes, "Operation scheduling of prosumer with renewable energy sources and storage devices," 2016 13th International Conference on the European Energy Market (EEM), Porto, 2016, pp. 1-5.

[2] R. Araneo and M. C. Falvo, "Simulation of a ESS in a prosumer powerplant with a PV system and an EV charging station," 2016 IEEE 16th International Conference on Environment and Electrical Engineering (EEEIC), Florence, 2016, pp. 1-5.

[3] M. C. Falvo, U. Grasselli, M. Manganelli and A. Modesto, "Small scale ESS for LV prosumers: An economic feasibility and sensitivity analysis," 2015 IEEE 15th International Conference on Environment and Electrical Engineering (EEEIC), Rome, 2015, pp. 950-955.

[4] G. Graditi, M.G. Ippolito, R. Rizzo, E. Telaretti, and G. Zizzo, "Technical-Economical Evaluations for Distributed Storage
Applications: An Italian Case Study for a Medium-Scale Public Facility". Paper presented at 3rd Renewable Power Generation Conference - RPG 2014, Napoli.

[5] Y. Wang, X. Lin and M. Pedram, "Adaptive Control for Energy Storage Systems in Households With Photovoltaic Modules," in IEEE Transactions on Smart Grid, vol. 5, no. 2, pp. 992-1001, March 2014.

[6] E. Romero-Cadaval, G. Spagnuolo, L. G. Franquelo, C. A. Ramos-Paja, T. Suntio and W. M. Xiao, "Grid-Connected Photovoltaic Generation Plants: Components and Operation," in IEEE Industrial Electronics Magazine, vol. 7, no. 3, pp. 6-20, Sept. 2013.

[7] M. J. E. Alam, K. M. Muttaqi and D. Sutanto, "Mitigation of Rooftop Solar PV Impacts and Evening Peak Support by Managing Available Capacity of Distributed Energy Storage Systems," in IEEE Transactions on Power Systems, vol. 28, no. 4, pp. 3874-3884, Nov. 2013.

[8] M. Sechilariu, B. Wang and F. Locment, "Building Integrated Photovoltaic System With Energy Storage and Smart Grid Communication," in IEEE Transactions on Industrial Electronics, vol. 60, no. 4, pp. 1607-1618, April 2013.

[9] H. Beltran, E. Bilbao, E. Belenguer, I. Etxeberria-Otadui and P. Rodriguez, "Evaluation of Storage Energy Requirements for Constant Production in PV Power Plants," in IEEE Transactions on Industrial Electronics, vol. 60, no. 3, pp. 1225-1234, March 2013.

[10] E. Romero-Cadaval, B. Francois, M. Malinowski and Q. C. Zhong, "Grid-Connected Photovoltaic Plants: An Alternative Energy Source, Replacing Conventional Sources," in IEEE Industrial Electronics Magazine, vol. 9, no. 1, pp. 18-32, March 2015.

[11] H. Zhou, T. Bhattacharya, D. Tran, T. S. T. Siew and A. M. Khambadkone, "Composite Energy Storage System Involving Battery and Ultracapacitor With Dynamic Energy Management in Microgrid Applications," in IEEE Transactions on Power Electronics, vol. 26, no. 3, pp. 923-930, March 2011.

[12] H. F. Gharibeh, A. S. Yazdankhah and M. R. Azizian, "Improved energy management for a power-split multi-source fuel cell vehicle based on optimal source sizing and regenerative braking," 2016 IEEE 16th International Conference on Environment and Electrical Engineering (EEEIC), Florence, 2016, pp. 1-6.

[13] M. Momayyezan, D. B. W. Abeywardana, B. Hredzak and V. G. Agelidis, "Integrated Reconfigurable Configuration for Battery/Ultracapacitor Hybrid Energy Storage Systems," in IEEE Transactions on Energy Conversion, vol. 31, no. 4, pp. 1583-1590, Dec. 2016.

[14] M. E. Glavin, P. K. W. Chan, S. Armstrong and W. G. Hurley, "A standalone photovoltaic supercapacitor battery hybrid energy storage system," 2008 13th International Power Electronics and Motion Control Conference, Poznan, 2008, pp. 1688-1695.

[15] C. R. Akli, X. Roboam, B. Sareni and A. Jeunesse, "Energy management and sizing of a hybrid locomotive," 2007 European Conference on Power Electronics and Applications, Aalborg, 2007, pp. 1-10.

[16] O. Palizban and K. Kauhaniemi, "Power sharing for distributed energy storage systems in AC microgrid: Based on state-of-charge," 2015 IEEE PES Asia-Pacific Power and Energy Engineering Conference (APPEEC), Brisbane, QLD, 2015, pp. 1-5.

[17] J. Zou, C. Peng, J. Shi, X. Xin and Z. Zhang, "State-of-charge optimising control approach of battery energy storage system for wind farm," in IET Renewable Power Generation, vol. 9, no. 6, pp. 647-652, 8 2015.

[18] H. Yin, W. Zhou, M. Li, C. Ma and C. Zhao, "An Adaptive Fuzzy Logic-Based Energy Management Strategy on Battery/Ultracapacitor Hybrid Electric Vehicles," in IEEE Transactions on Transportation Electrification, vol. 2, no. 3, pp. 300-311, Sept. 2016.

[19] M. Ruiz-Cortés, E. Romero-Cadaval, C. Roncero-Clemente, F. BarreroGonzález and E. González-Romera, "Energy management strategy to coordinate batteries and ultracapacitors of a hybrid energy storage system in a residential prosumer installation," 2017 International Young Engineers Forum (YEF-ECE), Costa da Caparica, Portugal, 2017, pp. 30-35. 